\title{
The suprachoroidal space: from potential space to a space with potential
}

Clinical Ophthalmology

25 January 2016

Number of times this article has been viewed

\author{
Elad Moisseiev ${ }^{1-3}$ \\ Anat Loewenstein ${ }^{2,3}$ \\ Glenn Yiu' \\ 'UC Davis Eye Center, University of \\ California Davis, Sacramento, CA, \\ USA; ${ }^{2}$ Ophthalmology Department, \\ Tel Aviv Medical Center, Tel Aviv, \\ ${ }^{3}$ Sackler School of Medicine, Tel Aviv \\ University, Tel Aviv, Israel
}

\begin{abstract}
Recent advances have made it possible to image the suprachoroidal space, and the understanding of its clinical applications is currently being greatly expanded. This opinion piece covers the advances in imaging techniques that enable the demonstration of the suprachoroidal space, and its implication in various retinal pathologies. It also reviews its potential uses as a route for drug delivery for the treatment of retinal diseases, and its use in innovative surgical techniques. Current research is leading the way for the suprachoroidal space to be an aspect of retinal disease diagnosis, monitoring, medical treatment, and surgical manipulation.
\end{abstract}

Keywords: suprachoroidal space, enhanced depth imaging, drug delivery, potential space

\section{Introduction}

The suprachoroidal space (SCS) has been long known as a potential space between the choroid and the sclera. While the inner border of the choroid, which is the Bruch's membrane, is compact, the outer border is more a zone of transition, consisting of several fibrous lamellae with variable thickness. ${ }^{1}$ Until recently, the SCS could only be visualized on histology, ${ }^{2}$ and could only be demonstrated in vivo by ultrasonography in pathologic conditions such as idiopathic uveal effusion syndrome or posterior scleritis where excess fluid accumulation around the optic nerve results in a "T sign". 3 Optical coherence tomography (OCT) imaging has revolutionized the practice of ophthalmology in the past 2 decades, mostly for retinal conditions, and enabled detailed in vivo imaging of retinal structures with an axial resolution as high as $3 \mu \mathrm{m} .{ }^{4}$ Further advances in OCT technology have made it possible to clearly image not only the retina, but the layers underneath it. This is achieved by using enhanced depth imaging (EDI)-OCT, a technique that allows standard spectral-domain OCT instruments to visualize the choroid, ${ }^{5}$ or by swept-source OCT, which uses a longer wavelength that allows deeper penetration. ${ }^{6}$

These advanced imaging techniques have increased our understanding of retinal pathologies, demonstrating changes in choroidal thickness in a variety of conditions, such as central serous chorioretinopathy, ${ }^{7-11}$ polypoidal choroidal vasculopathy, ${ }^{12-14}$ myopic degeneration, ${ }^{15-16}$ and age-related macular degeneration (AMD). ${ }^{17-19}$ Choroidal imaging has also provided new insight on eyes with diabetic retinopathy, adult vitelliform pattern dystrophy, Stargardt's disease, pseudophakic cystoid macular edema, and intraocular inflammation. ${ }^{20-24}$ They can be used to diagnose and monitor choroidal nevi and tumors, and even improve the detection of choroidal melanoma. ${ }^{25-29}$

Further advances have now made it possible to image the SCS in vivo (Figure 1) to reveal new insights into its role in healthy and pathologic eyes. The ability to accurately image the choroid has made it an integral component in the evaluation of retinal conditions, and we expect the same for the SCS. Importantly, the SCS may not only 

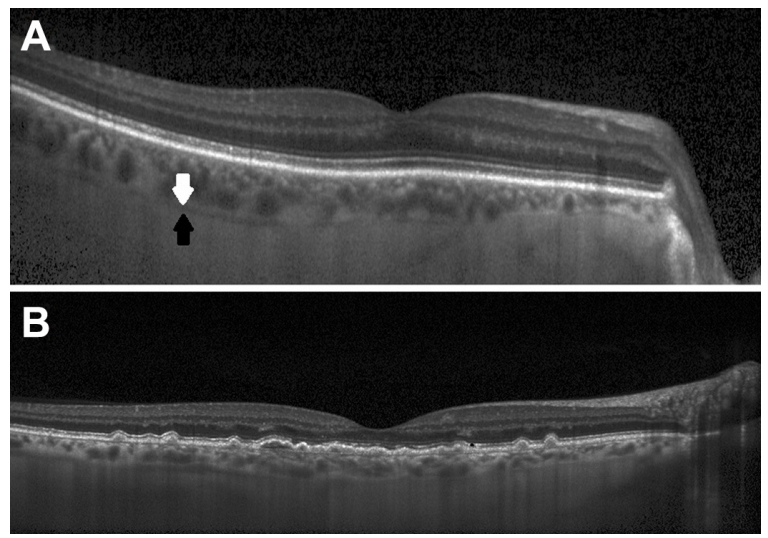

Figure I Imaging of the suprachoroidal space.

Notes: (A) An EDI-OCT scan of a healthy 67-year-old patient. The suprachoroidal space can be seen as a hyporeflective band between the outer border of the choroid (white arrow) and the inner border of the sclera (black arrow), which can be followed throughout the scan. (B) An EDI-OCT scan of a patient with age-related macular degeneration in which the suprachoroidal space can be seen.

Abbreviation: EDI-OCT, enhanced depth imaging-optical coherence tomography.

be useful for diagnosis and monitoring but may also provide a new route for both medical and surgical treatments. The purpose of this opinion piece is to focus on the SCS and its evolving relevance to retinal specialists.

\section{Imaging the SCS}

The majority of studies on choroidal thickness have defined the inner border of the sclera as the outer boundary of the choroid. However, some researchers have noted a hyporeflective band between the outer border of the choroid and sclera, which may cause variability in choroidal thickness measurements..$^{30}$ A recent study has identified this hyporeflective band to correspond to the SCS, and discussed the importance of its recognition to accurate measurement of choroidal thickness. The SCS can be demonstrated and accurately measured by both EDI-OCT and swept-source OCT, and has been shown to be present in approximately $50 \%$ of people above the age of 50 years. ${ }^{31,32}$ The presence of the SCS has been correlated to hyperopic refractive error, ${ }^{31}$ and is generally absent in the eyes of young, healthy persons. ${ }^{31,32}$ It has been theorized that in hyperopic eyes, there is increased hydrostatic pressure from compression of the vortex veins by the sclera, resulting in a subclinical suprachoroidal effusion that makes the small amount of fluid in the SCS visible on imaging. It has also been suggested that with age there is an increase in leakage of proteins from choroidal vessels to the SCS, increasing its osmotic pressure as well, and results in the higher rates of its detection in older individuals. ${ }^{31}$

The SCS has been reported to be demonstrated in $20 \%-50 \%$ of eyes with AMD. ${ }^{31-33}$ Except for the patients' increased age, it has been suggested that choroidal thinning in AMD may increase the likelihood of SCS's presence. ${ }^{33}$ Presence of SCS was also found in $50 \%-60 \%$ of patients with macular holes and epiretinal membranes,${ }^{32}$ but this may be due to their age and not necessarily correlated to these conditions. It has also been shown that there is loculation of fluid in the outer choroid in approximately $65 \%$ of eyes with central serous chorioretinopathy. ${ }^{34}$ While the presence or thickness of the SCS in retinal diseases has not been fully elucidated, it may become another parameter that will be useful for their diagnosis and follow-up.

\section{The SCS as a route for drug delivery}

Intravitreal injections are currently the mainstay of treatment for a wide variety of retinal diseases, and constitute an effective method to achieve high intraocular levels of antibiotic, antiviral, antifungal, steroidal, and anti-VEGF drugs. ${ }^{35,36}$ Intravitreal injections have a good safety profile, but it should be remembered that they can cause ocular complications, including cataract formation, glaucoma, choroidal hemorrhage, endophthalmitis, vitreous hemorrhage, and retinal detachment, ${ }^{37}$ and the injected drug can be absorbed and induce systemic adverse effects. Topical administration of drugs generally achieves low concentration in the vitreous, and is not commonly used for the treatment of retinal diseases. ${ }^{38}$ Systemic treatment is also limited, as the blood-retina-barrier limits the ability of the drugs to reach the eye, and high doses may cause systemic adverse effects. ${ }^{39}$ Periocular (subconjunctival, sub-Tenon, or retrobulbar) injections can bypass the blood-retina-barrier without intraocular penetration. However, the injected drug still has to traverse the sclera, which is less permeable to larger molecules, and may not achieve therapeutic drug levels at the level of the retina. $^{39,40}$

The SCS is an attractive route of drug delivery, which may enable bypassing the sclera without the risk of intraocular penetration. Animal studies have shown that the SCS can accommodate up to $1 \mathrm{~mL}$ of fluid, which rapidly diffused into the posterior segment. ${ }^{41}$ This volume is much larger than what is required for achieving therapeutic levels for clinically relevant drugs. Injections of $10-50 \mu \mathrm{L}$ into the SCS have been demonstrated to be well tolerated with a low risk of ocular complications. ${ }^{42,43}$ OCT imaging of the SCS may also become a useful means of ensuring the injected drug was properly administered, as well as of following its pharmacokinetics. ${ }^{42}$ Suprachoroidal drug delivery to the vitreous has been shown to decline with an increase in drug lipophilicity and molecular weight. ${ }^{44}$ This evidence 
makes the SCS an attractive route for drug delivery, as it may allow for larger volumes of drugs and longer duration of action to be achieved with a safer procedure. In addition, drug delivery through the SCS bypasses the internal limiting membrane barrier and outer blood-retina-barrier, and may be a preferred route for drug delivery targeting the retinal pigment epithelium.

Several techniques for injections into the SCS have been described. First, the injection can be done using standard small-gauge needles, but this is a delicate procedure and penetration into the choroid or the vitreous cavity is possible. Second, surgical cannulation has been shown to be effective and safe in achieving drug delivery to the posterior pole, ${ }^{45}$ but this is a complicated procedure that cannot be performed in-office. Finally, SCS drug delivery can be performed using microneedles - small-gauge needles ( $30-33 \mathrm{G})$ only $0.7-1.0$ $\mathrm{mm}$ in length that are only long enough to penetrate the sclera and reach the SCS. ${ }^{46}$ These microneedles have been demonstrated to be safe and effective in several animal studies. ${ }^{46-49}$ Specifically, administration of corticosteroids via this route was shown to reduce intraocular inflammation, ${ }^{48}$ and antiglaucoma agents were shown to reduce intraocular pressure. ${ }^{49}$ A microneedle for SCS injection in humans has been developed by Clearside Biomedical Inc. (Alpharetta, GA, USA), and a Phase $1 / 2$ study evaluating the safety and tolerability of $4 \mathrm{mg} / 100 \mu \mathrm{L}$ triamcinolone acetonide administered to the SCS in patients with noninfectious uveitis has been performed (clinicaltrials.gov NCT01789320; data not published yet). It is likely that in the near future, this will become a common route of drug delivery for the treatment of retinal diseases.

\section{Retinal surgery in the SCS}

Surgical utilization of the SCS has primarily been investigated for the treatment of glaucoma. This concept has been described over a century ago, when iatrogenic creation of a cyclodialysis cleft was performed to reduce intraocular pressure in patients with severe glaucoma, thus creating an alternate route of aqueous fluid passage into the SCS. ${ }^{50,51}$ Glaucoma surgery in the SCS has several important advantages over penetrating procedures. It can be entered by transscleral (external) or intracameral (internal) approaches, it does not disturb the conjunctiva or preclude subsequent surgeries, and has an increased safety profile. ${ }^{50,52,53}$ Several surgical techniques have been developed to use the SCS for glaucoma, such as a silicone drainage device that is implanted under a scleral flap and connects the anterior chamber and SCS, ${ }^{54,55}$ the Gold Micro Shunt which is implanted in the $\mathrm{SCS},{ }^{56}$ and several types of supraciliary microstents that are inserted through a clear corneal incision (often during cataract surgery) to create an internal connection from the anterior chamber to the SCS. ${ }^{57-59}$

The SCS has not been the target of vitreoretinal surgeons, and was likely only accessed by them in complicated cases requiring surgical drainage of suprachoroidal hemorrhage. However, as technology and instrumentations continue to evolve, it may become useful and important in vitreoretinal surgery. For instance, it has been shown that injection of sodium hyaluronate into the SCS in patients with retinal detachment achieved choroidal detachment that lasted long enough to allow successful retinopexy. ${ }^{60}$ More recently, a technique of suprachoroidal buckling has been described, using an illuminated catheter inserted into the SCS and navigated to any desired location, where a long-lasting hyaluronic acid filler can be injected to create internal choroidal indentation. This technique can be performed without or in combination with vitrectomy, and has been successfully used for the treatment of patients with retinal detachment and myopic tractional maculopathies. ${ }^{61-63}$ Although pars plana vitrectomy and scleral buckles are effective surgical techniques, they may still cause complications such as pain, diplopia, refractive error shifts, cataract progression, glaucoma, vitreous hemorrhage, and endophthalmitis. ${ }^{64}$ Suprachoroidal buckling can be an elegant and relatively noninvasive technique that may become a useful adjunct to vitreoretinal surgery, although the technical difficulty may limit its widespread use for now.

Another aspect where the SCS may prove to be useful is retinal prosthesis surgery. Retinal prostheses are electrical devices that can be implanted directly under or on top of the retina, and restore some vision in patients with severe visual loss. At present, several devices have been developed for this purpose, ${ }^{65}$ although only the Argus II system has been approved by the US Food and Drug Administration. Results from patients implanted with the Argus II demonstrated long-term efficacy and safety, ${ }^{66}$ and paved the way for further research into this field. Despite the encouraging results, it should be remembered that implantation of retina prostheses requires a complicated surgical procedure, that may be associated with significant complications. A retinal prosthesis that can be placed in the SCS would have the advantages of a simpler surgical procedure and increased safety profile. Recently, a prototype for a retinal prosthesis implanted in the SCS has been developed. The device consists of a conformable silicone substrate that holds 21 electrodes inserted into the SCS and placed under the macula. A wire exits through the sclera, and is passed 
through a tunnel dissected under the temporalis fascia to a percutaneous pedestal which is placed periauricularly. ${ }^{67}$ The device has been studied in cadaver and animal eyes, ${ }^{68}$ and recently reported to have resulted in some vision gain with a favorable safety profile in three human patients with end-stage retinitis pigmentosa. ${ }^{69}$ Further research into this type of retinal prosthesis is ongoing.

\section{Conclusion}

The SCS is receiving increasing attention, and its potential role in the diagnosis and treatment of various retinal conditions is being recognized. Technological advances have only recently made it possible for the SCS to be clearly imaged, and its variability in aging, health, and disease states is currently being understood. It is also emerging as a target for drug delivery and surgical procedures, spawning an array of novel devices for clinical use, from microneedles to retinal prostheses. It is likely that in the near future, the SCS will become a routine component in the diagnosis, monitoring, and treatment of retinal diseases, and it is likely that new discoveries regarding its potential uses will be made in the near future. In the meantime, we suggest clinicians try to identify and characterize the SCS in their patients' OCT scans, as awareness of it holds the promise for interesting and clinically significant insights.

\section{Disclosure}

The authors report no conflicts of interest in this work.

\section{References}

1. Buggage RR, Grossniklaus HE. Choroid and suprachoroid. In: Tasman W, Jaeger AE, editors. Duane's Foundations of Clinical Ophthalmology. Vol 1. Northwestern University: J.B. Lippincott: Philadelphia, PA, USA; 1991.

2. Krohn J, Bertelsen T. Corrosion casts of the suprachoroidal space and uveoscleral drainage routes in the human eye. Acta Ophthalmol Scand. 1997;75(1):32-35.

3. Benson WE. Posterior scleritis. Surv Ophthalmol. 1988;32(5): 297-316.

4. Ko TH, Fujimoto JG, Schuman JS, et al. Comparison of ultrahigh- and standard-resolution optical coherence tomography for imaging macular pathology. Ophthalmology. 2005;112(11):1922.e1-e15.

5. Spaide RF, Koizumi H, Pozzoni MC. Enhanced depth imaging spectral-domain optical coherence tomography. Am J Ophthalmol. 2008;146(4):496-500.

6. Michalewska Z, Michalewski J, Nawrocki J. Going deeper and going wider. Retinal Physician. 2013;3:42-48.

7. Hamzah F, Shinojima A, Mori R, Yuzawa M. Choroidal thickness measurement by enhanced depth imaging and swept-source optical coherence tomography in central serous chorioretinopathy. BMC Ophthalmol. 2014; 14:145.

8. Ferrara D, Mohler KJ, Waheed N, et al. En face enhanced-depth swept-source optical coherence tomography features of chronic central serous chorioretinopathy. Ophthalmology. 2014;121(3): 719-726.
9. Yang L, Jonas JB, Wei W. Optical coherence tomography-assisted enhanced depth imaging of central serous chorioretinopathy. Invest Ophthalmol Vis Sci. 2013;54(7):4659-4665.

10. Imamura Y, Fujiwara T, Margolis R, Spaide RF. Enhanced depth imaging optical coherence tomography of the choroid in central serous chorioretinopathy. Retina. 2009;29(10):1469-1473.

11. Maruko I, Iida T, Sugano Y, Ojima A, Sekiryu T. Subfoveal choroidal thickness in fellow eyes of patients with central serous chorioretinopathy. Retina. 2011;31(8):1603-1608.

12. Yang LH, Jonas JB, Wei WB. Optical coherence tomographic enhanced depth imaging of polypoidal choroidal vasculopathy. Retina. 2013;33(8):1584-1589.

13. Chung SE, Kang SW, Lee JH, Kim YT. Choroidal thickness in polypoidal choroidal vasculopathy and exudative age-related macular degeneration. Ophthalmology. 2011;118(5):840-845.

14. Jirarattanasopa $P$, Ooto $S$, Nakata I, et al. Choroidal thickness, vascular hyperpermeability, and complement factor $\mathrm{H}$ in age-related macular degeneration and polypoidal choroidal vasculopathy. Invest Ophthalmol Vis Sci. 2012;53(7):3663-3672.

15. Ho M, Liu DT, Chan VC, Lam DS. Choroidal thickness measurement in myopic eyes by enhanced depth optical coherence tomography. Ophthalmology. 2013;120(9):1909-1914.

16. Fujiwara T, Imamura Y, Margolis R, Slakter JS, Spaide RF. Enhanced depth imaging optical coherence tomography of the choroid in highly myopic eyes. Am J Ophthalmol. 2009;148(3):445-450.

17. Lindner M, Bezatis A, Czauderna J, et al. Choroidal thickness in geographic atrophy secondary to age-related macular degeneration. Invest Ophthalmol Vis Sci. 2015;56(2):875-882.

18. Yiu G, Chiu SJ, Petrou PA, et al. Relationship of central choroidal thickness with age-related macular degeneration status. Am J Ophthalmol. 2015;159(4):617-626.

19. Jonas JB, Forster TM, Steinmetz P, Schlichtenbrede FC, Harder BC. Choroidal thickness in age-related macular degeneration. Retina. 2014; 34(6):1149-1155.

20. Querques G, Lattanzio R, Querques L, et al. Enhanced depth imaging optical coherence tomography in type 2 diabetes. Invest Ophthalmol Vis Sci. 2012;53(10):6017-6024.

21. Puche N, Querques G, Blanco-Garavito R, et al. En face enhanced depth imaging optical coherence tomography features in adult onset foveomacular vitelliform dystrophy. Graefes Arch Clin Exp Ophthalmol. 2014;252(4):555-562.

22. Baltmr A, Lightman S, Tomkins-Netzer O. Examining the choroid in ocular inflammation: a focus on enhanced depth imaging. JOphthalmol. 2014;2014:459136.

23. Odrobina D, LaudaŃska-Olszewska I. Choroidal thickness in clinically significant pseudophakic cystoid macular edema. Retina. 2015;35(1):136-140.

24. Piri N, Nesmith BL, Schaal S. Choroidal hyperreflective foci in Stargardt disease shown by spectral-domain optical coherence tomography imaging: correlation with disease severity. JAMA Ophthalmol. 2015;133(4):398-405.

25. Dolz-Marco R, Hasanreisoglu M, Shields JA, Shields CL. Posterior scleral bowing with choroidal nevus on enhanced-depth imaging optical coherence tomography. JAMA Ophthalmol. 2015;133(10):1165-1170.

26. Shields CL, Manalac J, Das C, Saktanasate J, Shields JA. Review of spectral domain enhanced depth imaging optical coherence tomography of tumors of the choroid. Indian J Ophthalmol. 2015;63(2):117-121.

27. Shields CL, Kaliki S, Rojanaporn D, Ferenczy SR, Shields JA. Enhanced depth imaging optical coherence tomography of small choroidal melanoma: comparison with choroidal nevus. Arch Ophthalmol. 2012;130(7):850-856.

28. Torres VL, Brugnoni N, Kaiser PK, Singh AD. Optical coherence tomography enhanced depth imaging of choroidal tumors. Am J Ophthalmol. 2011;151(4):586-593.

29. Daitch Z, Shields CL, Say EA, Mashayekhi A, Shields JA. Submillimeter choroidal melanoma detection by enhanced depth imaging optical coherence tomography in a patient with oculodermal melanocytosis. Retin Cases Brief Rep. 2016;10(1):6-10. 
30. Rahman W, Chen FK, Yeoh J, Patel P, Tufail A, Da Cruz L. Repeatability of manual subfoveal choroidal thickness measurements in healthy subjects using the technique of enhanced depth imaging optical coherence tomography. Invest Ophthalmol Vis Sci. 2011;52(5):2267-2271.

31. Yiu G, Pecen P, Sarin N, et al. Characterization of the choroid-scleral junction and suprachoroidal layer in healthy individuals on enhanceddepth imaging optical coherence tomography. JAMA Ophthalmol. 2014;132(2):174-181.

32. Michalewska Z, Michalewski J, Nawrocka Z, Dulczewska-Cichecka K, Nawrocki J. Suprachoroidal layer and suprachoroidal space delineating the outer margin of the choroid in swept-source optical coherence tomography. Retina. 2015;35(2):244-249.

33. Kim JH, Chang YS, Kim JW, Lee TG, Lew YJ. Imaging Suprachoroidal Layer in Exudative Age-Related Macular Degeneration. Curr Eye Res. Epub 2015 Aug 13.

34. Spaide RF, Ryan EH Jr. Loculation of Fluid in the Posterior Choroid in Eyes With Central Serous Chorioretinopathy. Am J Ophthalmol. 2015; 160(6):1211-1216.

35. Peyman GA, Lad EM, Moshfeghi DM. Intravitreal injection of therapeutic agents. Retina. 2009;29(7):875-912.

36. Heimann H. Chapter 5. Intravitreal injections: Techniques and sequelae. In: Holz FG, Spaide RF, editors. Medical Retina (Essentials in Ophthalmology). 1st ed. Berlin: Springer-Verlag Berlin Heidelberg; 2007:67-87.

37. Prasad AG, Schadlu R, Apte RS. Intravitreal pharmacotherapy: applications in retinal disease. Compr Ophthalmol Update. 2007;8(5): 259-269.

38. Abdelkader H, Alany RG. Controlled and continuous release ocular drug delivery systems: pros and cons. Curr Drug Deliv. 2012;9(4): $421-430$

39. Rai Udo J, Young SA, Thrimawithana TR, et al. The suprachoroidal pathway: a new drug delivery route to the back of the eye. Drug Discov Today. 2015;20(4):491-495.

40. Geroski DH, Edelhauser HF. Transscleral drug delivery for posterior segment disease. Adv Drug Deliv Rev. 2001;52(1):37-48.

41. Seiler GS, Salmon JH, Mantuo R, Feingold S, Dayton PA, Gilger BC. Effect and distribution of contrast medium after injection into the anterior suprachoroidal space in ex vivo eyes. Invest Ophthalmol Vis Sci. 2011;52(8):5730-5736.

42. Gu B, Liu J, Li X, Ma Q, Shen M, Cheng L. Real-Time Monitoring of Suprachoroidal Space (SCS) Following SCS Injection Using Ultra-High Resolution Optical Coherence Tomography in Guinea Pig Eyes. Invest Ophthalmol Vis Sci. 2015;56(6):3623-3634.

43. Chen M, Li X, Liu J, Han Y, Cheng L. Safety and pharmacodynamics of suprachoroidal injection of triamcinolone acetonide as a controlled ocular drug release model. J Control Release. 2015;203:109-117.

44. Kadam RS, Williams J, Tyagi P, Edelhauser HF, Kompella UB. Suprachoroidal delivery in a rabbit ex vivo eye model: influence of drug properties, regional differences in delivery, and comparison with intravitreal and intracameral routes. Mol Vis. 2013;19:1198-1210.

45. Olsen TW, Feng X, Wabner K, et al. Cannulation of the suprachoroidal space: a novel drug delivery methodology to the posterior segment. Am J Ophthalmol. 2006;142(5):777-787.

46. Patel SR, Lin AS, Edelhauser HF, Prausnitz MR. Suprachoroidal drug delivery to the back of the eye using hollow microneedles. Pharm Res. 2011;28(1):166-176

47. Kim YC, Edelhauser HF, Prausnitz MR. Targeted delivery of antiglaucoma drugs to the supraciliary space using microneedles. Invest Ophthalmol Vis Sci. 2014;55(11):7387-7397.

48. Gilger BC, Abarca EM, Salmon JH, Patel S. Treatment of acute posterior uveitis in a porcine model by injection of triamcinolone acetonide into the suprachoroidal space using microneedles. Invest Ophthalmol Vis Sci. 2013;54(4):2483-2492.
49. Patel SR, Berezovsky DE, McCarey BE, Zarnitsyn V, Edelhauser HF, Prausnitz MR. Targeted administration into the suprachoroidal space using a microneedle for drug delivery to the posterior segment of the eye. Invest Ophthalmol Vis Sci. 2012;53(8):4433-4441.

50. Patrianakos TD. Anatomic and physiologic rationale to be applied in accessing the suprachoroidal space for management of glaucoma. J Cataract Refract Surg. 2014;40(8):1285-1290.

51. Heine L. Die cyclodialyse, eine neue glaukomoperation [Introduction of cyclodialysis in glaucoma]. Dtsch Med Wochenschr. 1905;31:824-826. German.

52. Kammer JA, Mundy KM. Suprachoroidal devices in glaucoma surgery. Middle East Afr J Ophthalmol. 2015;22(1):45-52.

53. Bailey AK, Sarkisian SR Jr, Vold SD. Ab interno approach to the suprachoroidal space. J Cataract Refract Surg. 2014;40(8):1291-1294.

54. Ozdamar A, Aras C, Karacorlu M. Suprachoroidal seton implantation in refractory glaucoma: a novel surgical technique. J Glaucoma. 2003;12(4):354-359.

55. Unal M, Kocak Altintas AG, Koklu G, Tuna T. Early results of suprachoroidal drainage tube implantation for the surgical treatment of glaucoma. J Glaucoma. 2011;20(5):307-314.

56. Melamed S, Ben Simon GJ, Goldenfeld M, Simon G. Efficacy and safety of gold micro shunt implantation to the supraciliary space in patients with glaucoma: a pilot study. Arch Ophthalmol. 2009;127(3): 264-269.

57. Hoeh H, Vold SD, Ahmed IK, et al. Initial Clinical Experience With the CyPass Micro-Stent: Safety and Surgical Outcomes of a Novel Supraciliary Microstent. J Glaucoma. 2016;25(1):106-112.

58. Malvankar-Mehta MS, Chen YN, Iordanous Y, Wang WW, Costella J, Hutnik CM. iStent as a Solo Procedure for Glaucoma Patients: A Systematic Review and Meta-Analysis. PLoS One. 2015;10(5): e0128146

59. Craven ER, Katz LJ, Wells JM, Giamporcaro JE; iStent Study Group. Cataract surgery with trabecular micro-bypass stent implantation in patients with mild-to-moderate open-angle glaucoma and cataract: two-year follow-up. J Cataract Refract Surg. 2012;38(8): 1339-1345

60. Poole TA, Sudarsky RD. Suprachoroidal implantation for the treatment of retinal detachment. Ophthalmology. 1986;93(11):1408-1412.

61. El Rayes EN, Oshima Y. Suprachoroidal buckling for retinal detachment. Retina. 2013;33(5):1073-1075.

62. El Rayes EN, Elborgy E. Suprachoroidal buckling: technique and indications. J Ophthalmic Vis Res. 2013;8(4):393-399.

63. El Rayes EN. Suprachoroidal buckling. Dev Ophthalmol. 2014;54: 135-146.

64. Sodhi A, Leung LS, Do DV, Gower EW, Schein OD, Handa JT. Recent trends in the management of rhegmatogenous retinal detachment. Surv Ophthalmol. 2008;53(1):50-67.

65. Chuang AT, Margo CE, Greenberg PB. Retinal implants: a systematic review. Br J Ophthalmol. 2014;98(7):852-856.

66. Ho AC, Humayun MS, Dorn JD, et al. Long-Term Results from an Epiretinal Prosthesis to Restore Sight to the Blind. Ophthalmology. 2015;122(8):1547-1554.

67. Saunders AL, Williams CE, Heriot W, et al. Development of a surgical procedure for implantation of a prototype suprachoroidal retinal prosthesis. Clin Experiment Ophthalmol. 2014;42(7):665-674.

68. Villalobos J, Fallon JB, Nayagam DA, et al. Cortical activation following chronic passive implantation of a wide-field suprachoroidal retinal prosthesis. J Neural Eng. 2014;11(4):046017.

69. Ayton LN, Blamey PJ, Guymer RH, et al. First-in-human trial of a novel suprachoroidal retinal prosthesis. PLoS One. 2014;9(112):e115239. 


\section{Publish your work in this journal}

Clinical Ophthalmology is an international, peer-reviewed journal covering all subspecialties within ophthalmology. Key topics include: Optometry; Visual science; Pharmacology and drug therapy in eye diseases; Basic Sciences; Primary and Secondary eye care; Patient Safety and Quality of Care Improvements. This journal is indexed on

Submit your manuscript here: http://www.dovepress.com/clinical-ophthalmology-journal
PubMed Central and CAS, and is the official journal of The Society of Clinical Ophthalmology (SCO). The manuscript management system is completely online and includes a very quick and fair peer-review system, which is all easy to use. Visit http://www.dovepress.com/ testimonials.php to read real quotes from published authors. 